\title{
In vivo confocal microscopy in fungal keratitis
}

\author{
Emmanuelle Brasnu, Tristan Bourcier, Bénédicte Dupas, Sandrine Degorge, Thibault Rodallec, Laurent \\ Laroche, Vincent Borderie, Christophe Baudouin
}

\begin{abstract}
Background: Fungal keratitis is a major blinding eye disease found throughout the world, particularly in developing countries. Given the recent increase in Fusarium keratitis infections in contact lens wearers owing to contact lens solutions, a warning was recently issued by the Food and Drug Administration, making it a public health concern in developed countries.

Objective: To show the advantages of in vivo confocal microscopy imaging using the Heidelberg Retina Tomograph II-Rostock Cornea Module (HRTII-RCM) in the early diagnosis of fungal keratitis.

Methods: HRTII-RCM confocal microscopy was performed on five patients presenting with fungal keratitis and on three donor corneas contaminated with Fusarium solani, Aspergillus fumigatus and Candida albicans.

Results: Direct microscopic evaluation of corneal smears and culture revealed the presence of $F$ solani in four cases and $C$ albicans in one case. HRTII-RCM examination of the infected patients and contaminated donor corneas revealed numerous high-contrast elements resembling Fusarium, Aspergillus hyphae or Candida pseudofilaments in the anterior stroma.

Conclusion: HRTII-RCM in vivo confocal microscopy is a new, non-invasive and rapid technique for the early diagnosis of fungal keratitis, showing high-resolution images resembling fungal structures in the early phase of the disease.
\end{abstract}

$\mathrm{F}$ ungal keratitis (FK) is a severe blinding eye disease as well as a major and increasing cause of ocular morbidity throughout the world. The poor prognosis is linked to clinical and microbiological diagnostic difficulties, severe complications and currently unsatisfactory treatments. ${ }^{1}$ The clinical features of FK are not specific, and clinically it resembles more common causes of infectious keratitis. As antifungal agents available today are mostly fungistatic, anatomic loss of the eye occurs in $9-26.3 \%$ of patients despite a prolonged course of toxic medical treatment. ${ }^{2}$ According to the US Centers for Disease Control and Prevention, it is now a public health concern in developed countries, with a recent increase in Fusarium keratitis infections associated with contact lens wearers using the ReNu with MoistureLoc contact lens cleaning solution (Rochester, New York, USA). ${ }^{3}$ Thus, this product was subsequently taken off the market, with the support of the US Food and Drug Administration. ${ }^{4}$ Our purpose was to show the advantages of in vivo confocal microscopy imaging using the Heidelberg Retina Tomograph II-Rostock Cornea Module (HRTII-RCM) in the early diagnosis of FK, allowing early initiation of treatment.

\section{MATERIALS AND METHODS}

Five patients presenting with clinical signs and symptoms of FK were examined using a new in vivo confocal microscope, the HRTII-RCM (Heidelberg Engineering, Heidelberg, Germany).
With the addition of the Rostock Cornea Module, the HRTII is converted to an in vivo confocal laser scanning microscope. Before microscopy, one drop of topical anaesthetic (oxybuprocaine chlorhydrate $1.6 \mathrm{mg} / 0.4 \mathrm{ml}$ ) and one drop of gel tear substitute (carbomer 0.2\%) were instilled in the lower conjunctival fornix. The $x-y$ position of the image and section depth were controlled manually. According to the type of lens used to perform the examination, two sizes of $384 \times 384$ pixel images were obtained: $300 \mu \mathrm{m} \times 300 \mu \mathrm{m}$ and $400 \mu \mathrm{m} \times 400 \mu \mathrm{m}$.

All patients underwent laboratory investigations after ophthalmic examination and confocal microscopy were completed: direct microscopic evaluation and corneal scraping culture.

For comparison with patient findings and validation of images obtained in vivo, confocal microscopy imaging using the HRTII-RCM was separately performed on three donor corneas from the Saint Antoine University Hospital Eye Bank, Paris, France, 2 days after contamination with Fusarium solani, Aspergillus fumigatus and Candida albicans, from three strains cultured in the Quinze-Vingts Hospital Microbiology Laboratory, Paris, France.

As HRTII-RCM confocal microscopy is routinely used for examination of ocular surface disorders, is non-invasive, painless and does not raise any risk of complication, ${ }^{5}$ the ethics committee of Paris 6 University had stated that exploration of the cornea using this technique did not require specific approval. Informed consent for the purpose of the examination was obtained from all subjects.

\section{RESULTS}

Our cohort was composed of four women and one man. Mean age was 45.2 years (range 24-68). All patients were regular bilateral contact lens wearers presenting with unilateral corneal symptoms (ocular pain, blurred vision). Average duration of symptoms was 9 days (range 2-21). In all patients, slit-lamp examination showed a unilateral corneal infectious ulcer (figs $1 \mathrm{~A}$ and 2A), with stromal reaction associated with conjunctival injection, and anterior chamber reaction up to $2^{+}$cells.

Laboratory investigations performed on the patients' corneal scrapings further confirmed the fungal infection in all cases. Direct microscopic evaluation of corneal smears and culture revealed the presence of Fusarium solani (fig 1B) in four cases and $C$ albicans in one case (fig 2B).

HRTII-RCM examinations were performed before obtaining the microbiological results. The four $F$ solani-infected patients' corneas revealed numerous high-contrast lines $200-300 \mu \mathrm{m}$ in length and 3-5 $\mu \mathrm{m}$ in width, with branches at $90^{\circ}$ angles in the anterior stroma resembling Fusarium hyphae (fig 1C,D).

HRTII-RCM examination of the $C$ albicans-infected patient's cornea revealed numerous high-contrast elongated particles measuring $10-40 \mu \mathrm{m}$ in length and $5-10 \mu \mathrm{m}$ in width in

Abbreviations: FK, fungal keratitis; HRTII-RCM, Heidelberg Retina Tomograph II-Rostock Cornea Module 

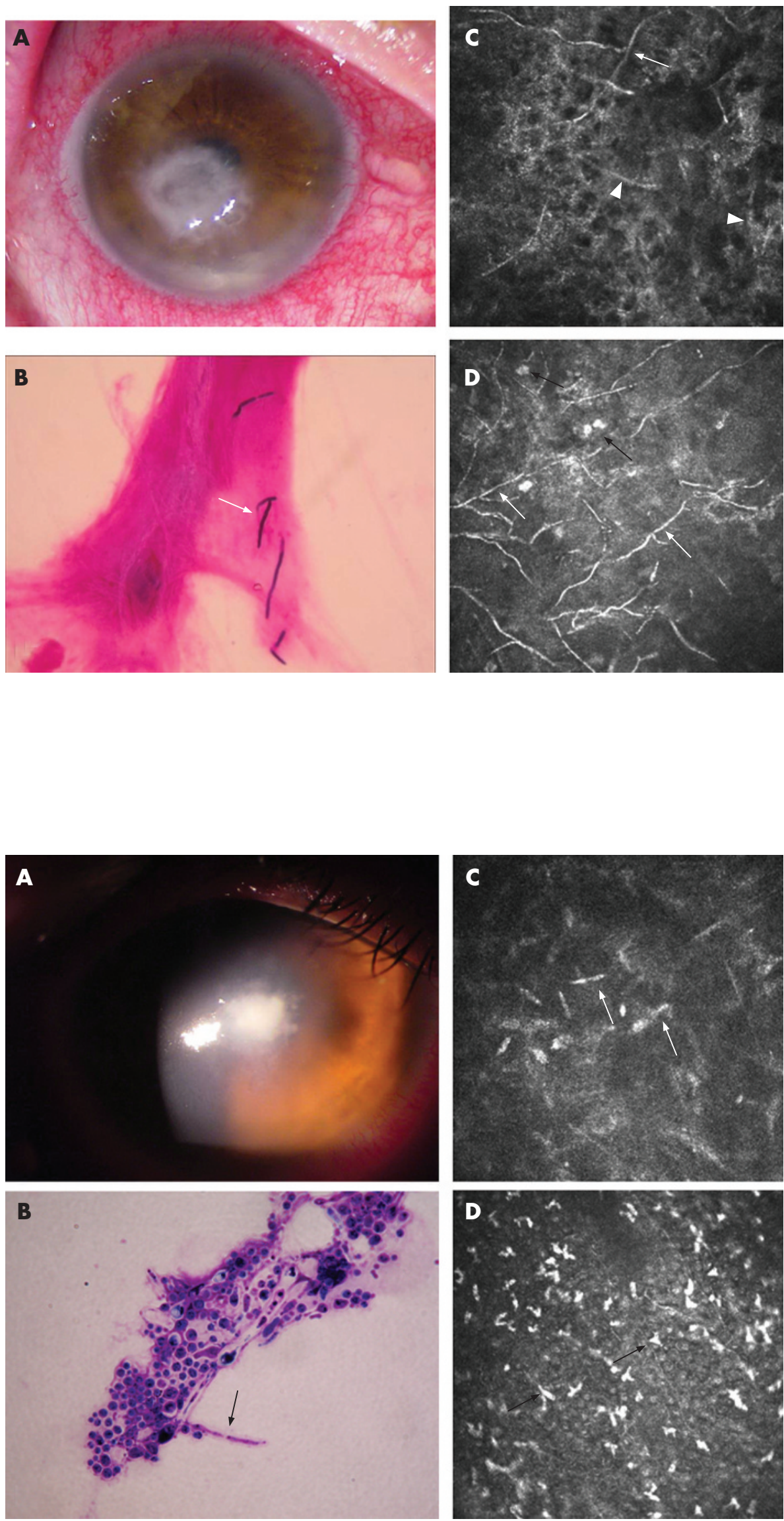

Figure 1 (A) Slit-lamp examination of a Fusarium solani-infected patient's cornea showing the corneal ulcer. (B) Direct microscopic evaluation of $F$ solani-infected patients' corneal smears (Gram staining; magnification $400 \times$ ) showing $F$ solani hyphae (arrow). (C, D) Heidelberg Retina Tomograph II-Rostock Cornea Module images $(300 \mu \mathrm{m} \times 300 \mu \mathrm{m})$ of two Fusarium solani-infected patients' corneas showing high-contrast lines resembling Fusarium hyphae (white arrows), high-contrast structures consistent with corneal nerves (arrowheads) and round inflammatory cells (black arrows).
Figure 2 (A) Slit-lamp examination of the Candida albicans-infected patient's cornea showing the corneal ulcer. (B) Cytological examination of the $C$ albicans-infected patients' corneal smears (May-GrunwaldGiemsa staining; magnification $1000 \times$ ) showing Calbicans pseudofilaments (arrow). (C,D) HRTII-RCM images $(400 \mu \mathrm{m} \times 400 \mu \mathrm{m})$ of the $C$ albicans-infected patient's cornea showing high-contrast elongated particles resembling Candida pseudofilaments (white arrows) and dendritiform inflammatory cells (black arrows). 

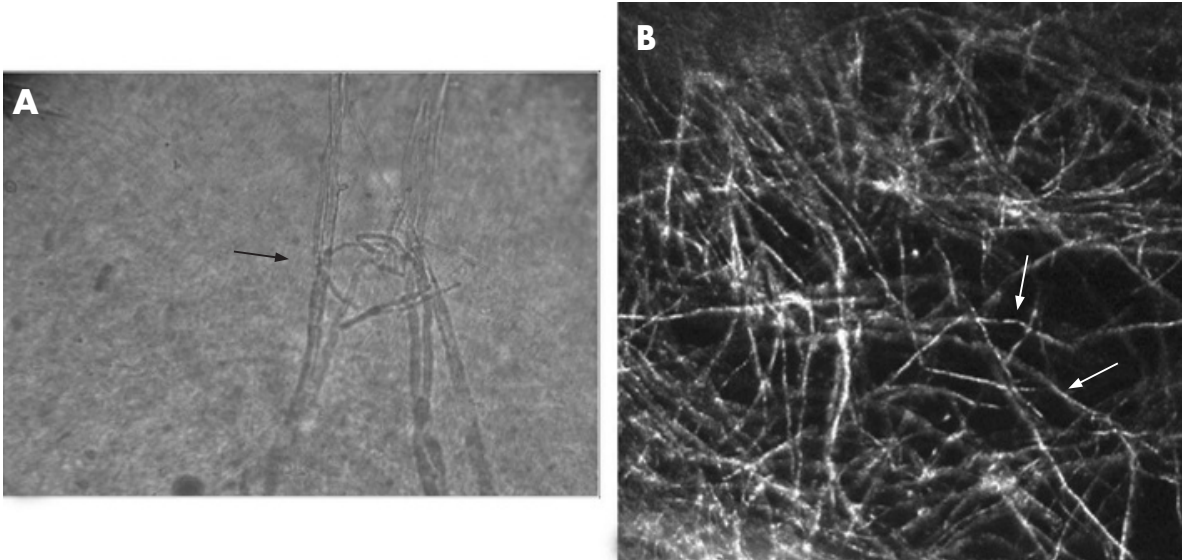

Figure 3 (A) Cytological examination (no staining, magnification $500 \times$ ) of the Fusarium solani-contaminated donor cornea showing Fusarium hyphae (arrow). (B) Heidelberg Retina Tomograph II-Rostock Cornea Module image (300 $\mu \mathrm{m} \times 300 \mu \mathrm{m})$ of the $F$ solani-contaminated donor cornea showing high-contrast lines resembling Fusarium hyphae (arrows)

the anterior stroma, resembling Candida pseudofilaments (fig 2C). In all cases, dendritiform and round inflammatory cells were present at the epithelial level and in the area of stromal infiltrates (figs 1D and 2D). Epithelial and stromal disorganisation was also observed, showing corneal fibroblast activation.

HRTII-RCM examination of the $F$ solani-contaminated donor cornea revealed numerous high-contrast lines 200-300 $\mu \mathrm{m}$ in length and 3-5 $\mu \mathrm{m}$ in width, with branches at $90^{\circ}$ angles resembling Fusarium hyphae and consistent with both the in vivo findings in infected patients and the cytological examination of corneal smears (fig 3A,B). HRTII-RCM examination of the $A$ fumigatus-contaminated donor cornea revealed numerous high-contrast lines 200-300 $\mu \mathrm{m}$ in length and 3-5 $\mu \mathrm{m}$ in width, with branches at $45^{\circ}$ angles resembling Aspergillus hyphae (fig 4A,B). HRTII examination of the $C$ albicans-contaminated donor cornea revealed numerous characteristic high-contrast elongated particles measuring $10-40 \mu \mathrm{m}$ in length and 5$10 \mu \mathrm{m}$ in width, consistent with Candida pseudofilaments (fig 4C,D).

\section{DISCUSSION}

Owing to the non-specific clinical features of FK, clinical diagnosis is difficult in the early phase of the disease, even with a high index of suspicion (history of corneal trauma, contact lens wear, topical steroid use). Laboratory diagnostic methods (direct microscopic evaluation, PCR and culture of corneal scrapings or biopsy specimens) are invasive and have a variable degree of sensitivity. ${ }^{1}$ Furthermore, approximately one-fourth of fungal cultures become positive only after 2 weeks. ${ }^{6}$
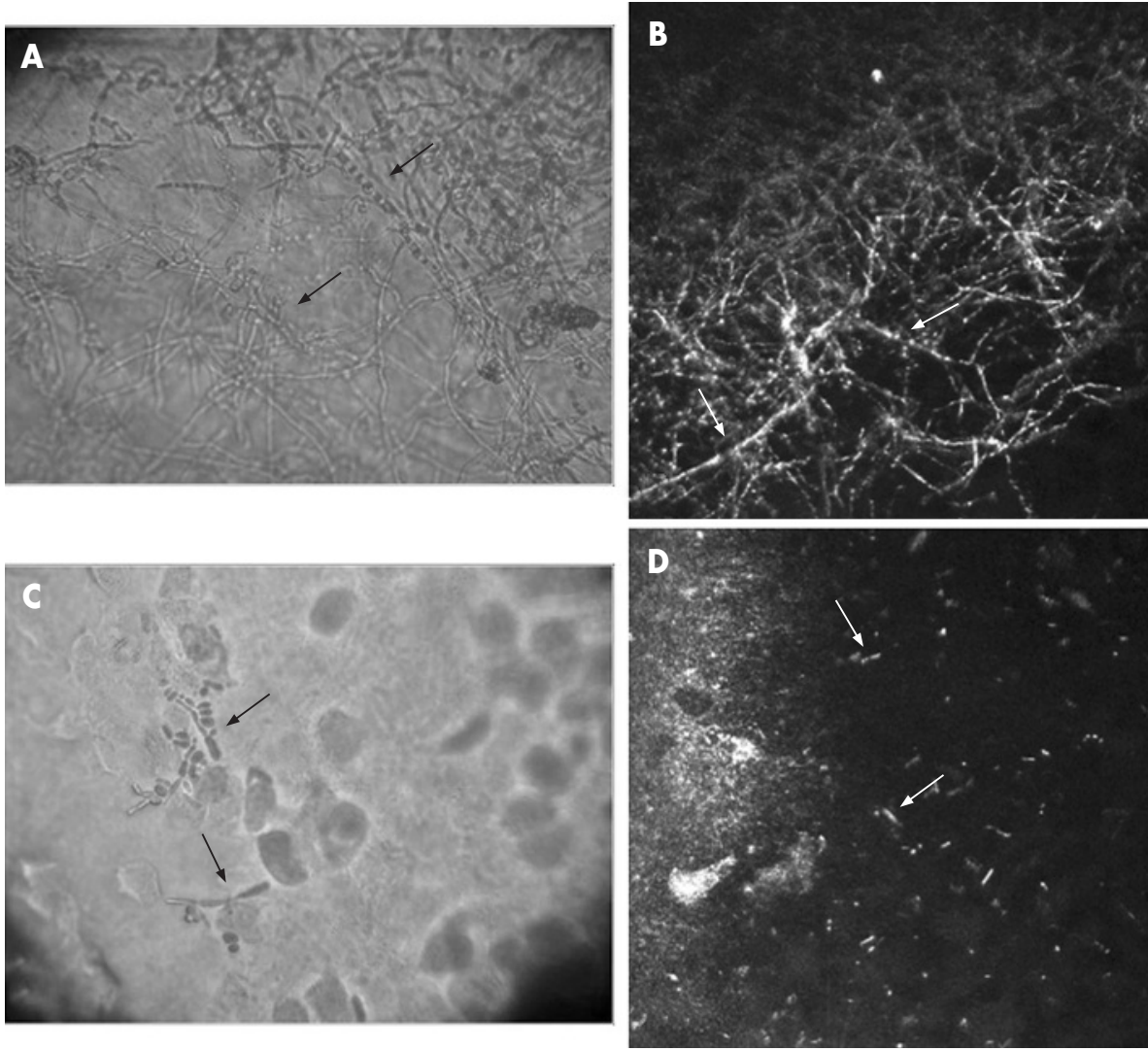

Figure 4 (A) Cytological examination (no staining, magnification $400 \times$ ) of the Aspergillus fumigatus-contaminated donor cornea showing Aspergillus hyphae (arrows). (B) Heidelberg Retina Tomograph II-Rostock Cornea Module image $(300 \mu \mathrm{m} \times 300 \mu \mathrm{m})$ of the $A$ fumigatuscontaminated donor cornea showing highcontrast lines resembling Aspergillus hyphae (arrows). (C) Cytological examination (no staining; magnification $400 \times$ ) of the Candida albicans-contaminated donor cornea showing Candida pseudofilaments (arrows). (D) HRTII-RCM image $(300 \mu \mathrm{m} \times 300 \mu \mathrm{m})$ of the $C$ albicanscontaminated donor cornea showing highcontrast elongated particles resembling Candida pseudofilaments (arrows). 
Therefore, FK is frequently misdiagnosed or diagnosed only at a very late stage, after aggressive treatments for bacterial or viral keratitis, or both, have failed. This delay in diagnosis and treatment can result in an irreversible, but possibly avoidable, loss of vision. Non-invasive, reliable and rapid diagnostic tools such as in vivo confocal microscopy may aid in saving useful vision in many eyes with FK.

In vivo first-generation confocal microscopy techniques have already been used in diagnoses of acanthamoeba and fungal keratitis..$^{7-11}$ Recently, Babu K and Murthy ${ }^{12}$ reported a case in which the HRTII-RCM new-generation confocal microscope was used in the diagnosis of a combined fungal and acanthamoeba keratitis. Like the first-generation confocal microscopes, HRTII-RCM provides non-invasive, high-contrast, in vivo images of the cornea at different depths from epithelium to endothelium. Images of fungal structures are obtained immediately and allow early treatment to be started, before laboratory investigations conclude on the definitive diagnosis.

Compared with the first-generation confocal microscopes, the HRTII-RCM has the additional advantages of facilitating the study of epithelial tissues and peripheral structures of the cornea and providing images of much higher resolution of the corneal structures. Its magnification $(800 \times)$ is higher than what was possible with the first-generation confocal microscopes $(380 \times) \cdot{ }^{13}$ This amount of magnification is high enough to visualise individual cells, including fungal hyphae and yeast, in the cornea. The high resolution of the new-generation confocal microscopes-that is, close to $1 \mu \mathrm{m}$-also improves the sensitivity of this technique to include visualisation of yeast, which has never been reported with first-generation microscopes.

In our study, the patients' images were compared with images of Fusarium hyphae and Candida pseudofilaments on infected donor corneas, with a strong resemblance between them, further validating our in vivo findings.

The advantage of HRTII-RCM in the early diagnosis of infectious corneal disease has already been described in acanthamoeba keratitis, identifying acanthamoeba cyst-like structures in the cornea. ${ }^{14}$ In studies of human keratitis, roughly one-third of all corneal ulcers are culture negative. ${ }^{15}$ Thus, the new-generation confocal microscopes now available might be extremely useful in the management and prognosis of many corneal ulcers, helping in the differential diagnosis between keratomycosis and acanthamoeba keratitis in the early phase of these diseases.

FK is a major ophthalmological problem in agriculture-based geographical regions with hot, humid, tropical and subtropical climates. It has a high prevalence in Asia (South India, China, Bangladesh, Nepal) and also in Ghana and South Florida, where filamentous fungi are implicated as major pathogens. ${ }^{16-18}$ In regions with temperate climates such as Britain, France and the Northern US, the incidence of FK remains low, and yeast, particularly Candida species, are the most common cause. ${ }^{1} 1920$ However, according to the Centers for Disease Control and Prevention, FK is increasing in frequency in industrialised countries where it is becoming a public health problem. ${ }^{3}$ HRTIIRCM may be helpful in managing this infection, providing an earlier diagnosis and earlier empirical treatment, which may lead to a better prognosis.

In conclusion, HRTII-RCM in vivo confocal microscopy is a new, non-invasive and rapid technique for the early diagnosis of FK. Showing high-resolution images of the fungal structures at the early phase of the disease, it allows early specific treatment, which may improve the functional outcome in this often severe disease, no longer limited to developing countries.

\section{Authors' affiliations}

Emmanuelle Brasnu, Bénédicte Dupas, Department of Ophthalmology III, Quinze-Vingts National Center of Ophthalmology, Paris, France

Tristan Bourcier, Department of Ophthalmology, Strasbourg Universitary Hospital, Strasbourg, France

Sandrine Degorge, Quinze-Vingts National Center of Ophthalmology, Laboratory, Paris, France

Thibault Rodallec, Department of Ophthalmology II, Quinze-Vingts

National Center of Ophthalmology, Paris, France

Laurent Laroche, Vincent Borderie, Department of Ophthalmology V, Quinze-Vingts National Center of Ophthalmology, Paris, France

Christophe Baudouin, Quinze-Vingts National Center of Ophthalmology, Department of Ophthalmology III, Paris, France; INSERM, UMR S 872,

Cordeliers Biomedical Institute, Paris Descartes and Pierre et Marie Curie Universities, Paris, France

Competing interests: None.

Correspondence to: Professor C Baudouin, Hôpital des Quinze-Vingts, Service III, 28 rue de Charenton, 75012 Paris, France; baudouin@quinzevingts.fr

Accepted 7 November 2006

Published Online First 6 December 2006

\section{REFERENCES}

1 Srinivasan M. Fungal keratitis. Curr Opin Ophthalmol 2004;15:321-7.

2 Garg P, Gopinathan U, Choudhary K, et al. Keratomycosis: clinical and microbiologic experience with dematiaceous fungi. Ophthalmology, 2000;107, 574-80.

3 Chang DC, Grant GB, O'Donnell K, et al. Multistate outbreak of Fusarium keratitis associated with use of a contact lens solution. JAMA 2006;296:953-63.

4 US Food and Drug Administration Statement: Bausch \& Lomb Global Recall of ReNu with MoistureLoc Contact Lens Cleaning Solution. http://www.fda.gov/ bbs/topics/NEWS/2006/NEW01371.html (accessed 15 May 2006).

5 Labbe A, Dupas B, Hamard P, et al. In vivo confocal microscopy study of blebs after filtering surgery. Ophthalmology 2005;112:1979.

6 O'Day DM, Akrabawi PL, Head WS, et al. Laboratory isolation techniques in human and experimental fungal infections. Am J Ophthalmol 1979;87:688-93.

7 Avunduk AM, Beuerman RW, Varnell ED, et al. Confocal microscopy of Aspergillus fumigatus keratitis. Br J Ophthalmol 2003:87:409-10.

8 Chew SJ, Beuerman RW, Assouline M, et al. Early diagnosis of infectious keratitis with in vivo real time confocal microscopy. CLAO J 1992;18:197-201.

9 Parmar DN, Awwad ST, Petroll WM, et al. Tandem scanning confocal corneal microscopy in the diagnosis of suspected acanthamoeba keratitis. Ophthalmology 2006;113:538-47

10 Winchester K, Mathers WD, Sutphin JE. Diagnosis of Aspergillus keratitis in vivo with confocal microscopy. Cornea 1997;16:27-31.

11 Florakis GJ, Moazami G, Schubert H, et al. Scanning slit confocal microscopy of fungal keratitis. Arch Ophthalmol 1997;115:1461-3.

12 Babu K, Murthy KR. Combined fungal and acanthamoeba keratitis: diagnosis by in vivo confocal microscopy. Eye 2007;21:271-2.

13 Stave J, Zinser G, Grummer G, et al. Modified Heidelberg retinal tomograph HRT. Initial results of in vivo presentation of corneal structures. Ophthalmologe 2002;99:276-80.

14 Bourcier T, Dupas B, Borderie V, et al. Heidelberg retina tomograph II findings of Acanthamoeba keratitis. Ocul Immunol Inflamm 2005;13:487-92.

15 McDonnell PJ, Nobe J, Gauderman WJ, et al. Community care of corneal ulcers. Am J Ophthalmol 1992;114:531-8.

16 Bharathi MJ, Ramakrishnan R, Vasu S, et al. Epidemiological characteristics and laboratory diagnosis of fungal keratitis. A three-year study. Indian J Ophthalmol 2003;51:315-21

17 Gopinathan U, Garg P, Fernandes M, et al. The epidemiological features and laboratory results of fungal keratitis: a 10-year review at a referral eye care center in South India. Cornea 2002;21:555-9.

18 Leck AK, Thomas PA, Hagan M, et al. Aetiology of suppurative corneal ulcers in Ghana and south India, and epidemiology of fungal keratitis. $\mathrm{Br} J$ Ophthalmol 2002;86:1211-15.

19 Thomas PA. Fungal infections of the cornea. Eye 2003;17:852-62.

20 Asbell P, Stenson S. Ulcerative keratitis. Survey of 30 years' laboratory experience. Arch Ophthalmol 1982;100:77-80. 